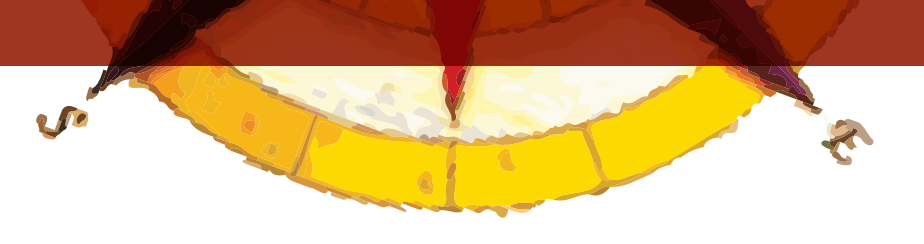

\title{
TOPOPHILIA AND PLACE EXPERIENCE
}

\author{
Professor Yi Fu Tuan ${ }^{1}$
}

\section{INTRODUCTION}

Academic spaces of Regional Dynamics and Semiotics and Aesthetic Thought, developed under the program of Bachelor in Elementary Education with Emphasis on Social Sciences of Universidad Distrital Francisco José de Caldas and the Research Geopaideia Group, interested in providing valuable elements in the discussion around current forms as geographical space is revealed and taught in our societies and educational contexts, especially in a teacher training program, it makes a bid to create a space for dialogue, taking advantage of recent digital media and communication, with experts in concepts and diverse epistemologies about geographic space and its significance in the world of life: it is how it is possible to consolidate an encounter with the teacher - Yi Fu Tuan. This meeting was primarily a time to explore emotional responses and for personal, academic and research enrichment full of opportunities for talking to and from the knowledge of Professor Tuan, about space issues.

The work of Professor Tuan, widely known to geographers, geography teachers and those interested in related issues has provided in recent decades valuable items for the understanding, study, research and experience of space from other possibilities in which the subject is a main character.

The Group Geopaideia aims to provide references for reflection in order to transform the traditional conception of geography in school that locates it only as a science for the enumeration and description of the biophysical phenomena on Earth's surface, being the geography neglected and marginalized as a task of just mechanical memorization of dates and locations forgetting its core of study which is the geographical and socio-cultural dimension.

This view is reinforced both in programs and curricula as well as in the imaginary social reference of the people who settled it in as the "science" in charge of the description and mapping, thus "The teaching of social sciences, particularly geography, in elementary school has been the target of a series of controversies in which people doubt the importance of this area in the training of learners, in their effectiveness and contribution that it provides to society and to the space in which they live "(Rodríguez, 2000:11).

It is needed to think of a different teaching, different than just a spatial one, as well as it is important to project learning opportunities that may arise in educational settings in order to advance in the understanding and experience of space in geographical education. "Education could provide a window into the consciousness of everyday spatial experiences. A geographical education of this nature could be part of the socialization process of individuals in a broad sense: as language is learnt, to recognize the codes and social settings and to communicate with others, only in this way we could aspire to a spatial socialization" (Lindón, 2009: 161).

1 Guest Professor of Geography, University of Wisconsin-Madison. 
This is the invitation and the challenges that we extend to all persons interested in a renewed geographical education, to the research projects, to the academic community in general and to our students, teachers in training. We hope that new generations of children and young people seize an education from and for spatiality.

Nubia Moreno Lache

\section{TOPOPHILIA AND PLACE EXPERIENCE}

I am very happy to participate in this video-conference. What I propose to do is to talk briefly about humanistic geography. My task will be all the easier if you in the audience have read the book Topophilia.

The book was written in 1974, thirty-five years ago, and since then I have moved on to other topics, though many draw on this early book. Topophilia is a novelty in geography because it recognizes human feelings and emotions. Strange that we geographers should have so consistently ignored them, since they are clearly a part of our everyday life. Each time we go to a place, especially to a new place, the emotional aspect of our being is aroused. Filling a glaring gap in the geographical literature inspired me to write Topophilia.

A century or so ago, geography was largely physical geography. When human beings were introduced, they were treated as though they were physical agents, like water, wind, and ice, with the power to change the face of our planet. A little later, say, early in the twentieth century, geographers introduced the concept of culture. We were not just physical agents, we were also cultural agents. Our culture influences greatly how we use our power to modify the Earth.

Still later, from the 1960s onward, another dimension was introduced into human geography--the psychological. Humans are psychological beings. They have moods and emotions, they perceive and experience in unique ways, depending on the acuteness of their sensory equipment, on culture, and on the physical environment itself. Let me take up the last by comparing the desert with the rain forest, the one open, the other enclosed. In the desert one can see great distances and it is difficult to be lost, for there is always the sun during the day and the stars at night to guide one's navigation. Not so, obviously, in the rain forest, one can never see far in cluttered environment, and, in the absence of prominent landmarks and stellar guidance, it is easy to be lost in it. I like the desert and detest the rain forest: that's my own personal psychological response. The contrast between desert and rain forest is extreme. Less extreme is the contrast between the landscape of Bolivia and that of China. Nonetheless, the differences that exist impact perception and experience.

Lastly, human beings are moral agents. Human geography becomes humanistic geography when not only the cultural and the psychological, but the moral dimension is introduced. Human geography tends to focus on the visible and the tangible. It maps houses, shops, and streets, population and highway traffic, and such like. It is also concerned with social relationships, how members of a community relate to one another. But it seldom explicitly addresses morality, whether, for instance, a particular set of relationship is moral or merely self-serving. The quality of life, a topic of deep concern to humanistic geographers, requires that we examine the perceptual and experiential qualities of a physical setting. Does the sidewalk cafe promote conversation? Does a city park exude an air of calm? Are the markets and shopping centers lively and yet not disorienting? Some of the 
desirable qualities are physical (are there enough places for people to sit?) and some of them are aesthetic, but some verge on the moral: for instance, does a park exude serenity? Are the benches so arranged that they encourage conversation among mothers as their young children run about, exercising their sturdy limbs?

A challenge to unveiling the moral is that one can't judge by looking at the physical environment or outward behavior alone. For example you see a man and a woman entering a drugstore. They buy what they need, and their outward behavior seems wholly appropriate to the environment of the drugstore. Both might stand next to the newsstand and glance at the headlines and both might pick up a soft drink from the dispenser as they wait. However, the man was buying medicine for his sick wife, whereas the woman was buying rat poison with the intention of murdering her husband. Behavior of man and woman inside the drugstore is almost identical, but the emotion inside each is totally different.

\section{TOPOFILIA Y LA EXPERIENCIA DEL LUGAR}

\section{Profesor Yi Fu Tuan ${ }^{2}$}

\section{INTRODUCCIÓN}

Los espacios académicos de Dinámicas Regionales y Semiótica y Pensamiento Estético desarrollados en el marco del programa de Licenciatura en Educación Básica con Enfasis en Ciencias Sociales de la Universidad Distrital Francisco José de Caldas y el Grupo Interinstitucional de Investigación Geopaideia, interesados por aportar elementos valiosos en la deliberación sobre las formas actuales como el espacio geográfico se devela y enseña en nuestras sociedades y contextos educativos, máxime en un programa de formación de docentes, hace una apuesta para generar un espacio de diálogo, aprovechando los recientes medios digitales y de la comunicación, con expertos en concepciones y epistemologías diversas sobre el espacio geográfico y su significación en el mundo de la vida; es así como se logra consolidar un escenario de encuentro con el maestro Yi - Fu Tuan. Encuentro que es ante todo un momento emotivo y de enriquecimiento personal, académico, investigativo y de posibilidades por conversar con y desde el saber del profesor Tuan alrededor de los asuntos espaciales.

La obra del profesor Tuan ampliamente conocida por los geógrafos, docentes de geografía e interesados en temas afines, ha brindado en las últimas décadas elementos valiosos en la comprensión, estudio, investigación y vivencia del espacio desde otras posibilidades y en las que el sujeto es actor principal.

El Grupo Geopaideia busca aportar referentes de reflexión para poder transformar la concepción tradicionalista de la geografía escolar y que la ubica únicamente como una ciencia encargada de la enumeración y descripción de los fenómenos biofísicos en la superficie terrestre, siendo relegada y marginada a una labor netamente de memorización mecánica de datos y lugares olvidando su centro de estudio que es el espacio geográfico y su dimensión socio cultural. 
Esta concepción es reafirmada tanto en programas y currículos como también en el imaginario y referente social de las personas que la instauran como la "ciencia" encargada de la descripción y elaboración de mapas; de este modo "La enseñanza de las Ciencias Sociales, particularmente de la geografía, en la educación básica ha sido blanco de una serie de polémicas en la que se duda de la importancia de esta área en la formación de los educandos, en su eficacia y en el aporte que proporciona a la sociedad y al espacio en que se vive" (Rodríguez; 2000:11).

Es necesario pensar una enseñanza diferente de lo espacial, así como es importante proyectar posibilidades didácticas que puedan plantearse en los escenarios educativos para poder avanzar en la comprensión y vivencia del espacio en la educación geográfica. "La educación podría constituirse en una ventana hacia la toma de conciencia de las experiencias espaciales cotidianas. Una educación geográfica de esta naturaleza podría ser parte de los procesos de socialización de los individuos en sentido amplio: así como se aprende el lenguaje, a reconocer los códigos y marcos sociales y a comunicarse con los otros, podríamos aspirar a una socialización espacial" (Lindón; 2009: 161).

Esta es la invitación y los retos que extendemos a todas las personas interesadas en una educación geográfica renovada, a los proyectos de investigación, a la comunidad académica en general y a nuestros estudiantes, docentes en formación. Esperamos que las nuevas generaciones de niños, niñas y jóvenes aprehendan una educación desde y para la espacialidad.

Nubia Moreno Lache

\section{TOPOFILIA Y EXPERIENCIA DEL LUGAR}

Estoy muy feliz de estar con ustedes, de acompañarlos en esta conferencia en las que les voy va a hablar sobre geografía humanística, espero que la mayoría de los estudiantes hayan leído el libro Topofilia.

El libro fue escrito en 1974, hace 35 años y desde entonces he tenido tiempo para darle importancia a todo lo que escribí. Topofilia está basado en las emociones, está basado mucho en lo que sentimos sobre todo cuando llegamos a un lugar por primera vez. Eso es lo que me inspiró para escribir el libro.

La geografía humanística ha dado unos giros enormes desde hace 100 años. Los geógrafos veían y trataban a los otros humanos como si fueran partes de nuestra geografía física, como si fueran ríos, montañas, como si fueran parte de la superficie de nuestro planeta. Es decir a los humanos no solo los veían como algo físico sino como seres únicamente físicos, como acontecía en el siglo XIX.

Sin embargo en la segunda mitad del siglo XX hay un cambio significativo en el mundo académico y eso toca también a la geografía, así que la forma como cambia la concepción depende en gran medida por la cultura, porque ya no nos ven solo como algo físico sino como unos seres culturales. Y es aquí en donde discursos como el de la percepción toman fuerza, no solo por lo que plantean, sino porque la percepción cambiaba y dependía mucho de la cultura que traían los humanos.

Dependía también de la cultura del lugar geográfico donde vivían. Si tu cultura era boliviana pues influía, si la cultura era china influía. La cultura era muy importante porque jugaba con unos libretos culturales que creaban otro tipo de ser humano en la geografía. 
Con el tiempo se añadió otra nueva dimensión, y fue la dimensión física, pero no la física de lo biofísico sino la parte física del ser; es decir se empezó a separar al ser en dimensiones. Así pues, comenzamos como algo físico, cultural, psicológico, como también la parte visual, la parte estética, pero quizá la más importante es la dimensión de la moralidad y las éticas.

Situación que es compleja porque se trata de jugar a comprender entre el ser ético, el ser cultural, el ser físico, el ser sujeto, pero también ser parte de un espacio, y ahí es donde hay una complicación muy fuerte para esa concepción geográfica diferente frente a una geografía fría de los geógrafos.

Hay una realidad invisible que no está a los ojos y que hace que el ser sea mucho más complejo desde lo emocional, desde lo emotivo, desde su aspecto ético y moral que aquello que podemos apreciar, y es así como empieza a concebirse el espacio físico en otra dimensión; es decir, también es incluirle al espacio una connotación ética y moral.

Aún así, a los geógrafos les ha preocupado mucho más la parte física de cómo lucen las ciudades, cómo son construidas, como son diseñadas y también la parte rural del campo, olvidando que la geografía humana se trata más del ser humano en su propia esencia. Justo allí es en donde juega un papel relevante la geografía humana, que va mucho más allá de la parte física, donde entran todas las dimensiones que nos constituyen.

Al igual que en el ser humano muchas veces vemos los comportamientos visibles, pero que no sabemos que es lo que está pasando por dentro, de la misma manera los geógrafos deben no solo preocuparse por diseñar calles o por diseñar tiendas sino por tratar de ser lo suficientemente sensibles para identificar el ambiente y la atmósfera que se puede crear entre ellos y la manera como nos podemos sentir dentro de estos lugares.

Por ejemplo, en América los geógrafos diseñan las calles, diseñan las ciudades, diseñan las tiendas, pero poco piensan sobre la forma como las personas, que son quienes las ocupan, visitan y emplean, las sienten, percibe, necesitan. En otras palabras, es importante también que los geógrafos piensen sobre cómo nos sentimos en los lugares y eso ha hecho mucha falta en la geografía humana tradicional.

Es necesario entonces también pensar en los lugares y en la forma como las personas representan dichos lugares; es decir, se está pensando el espacio fuera del sujeto, sería necesario volver a pensarlo dentro del sujeto. Quizá por ello es que de un momento a otro los lugares quedan fríos construidos por unos geógrafos expertos exteriores que planifican, diseñan, organizan, pero se les olvida una parte esencial que es el ser humano que habita el espacio.

Sin embargo, hay algo más complicado y está en nosotros mismos. Es muy difícil para nosotros encontrar una correlación entre nosotros y el medio ambiente. Por ejemplo, puedes observar dos personas que van a la droguería; una persona compra una medicina particular y la otra persona compra otro tipo de droga. Es perfectamente aceptable en una droguería; la gente va a comprar medicinas. Pero podría ser que la primera persona que compró la medicina compró la medicina para su esposa enferma y la segunda persona cuando entra a la droguería a comprar su medicina es para matar a su esposo. Su comportamiento dentro de la tienda es exactamente el mismo, pero la parte emocional dentro de cada uno de ellos es completamente diferente. Ese es también un asunto de la espacialidad, es decir, la intencionalidad con la que se crean y construyen las cosas están ante todo permeadas por la forma como los seres las viven, experimentan, sienten, emocionan, etc. El com- 
portamiento del hombre y la mujer dentro de la farmacia es casi idéntico, pero la emoción dentro de cada uno es totalmente diferente.

\section{BIBLIOGRAFÍA}

Lindón A. (2009) La espacialidad como fuente de las innovaciones de la vida cotidiana. En Lindón A. et al., La vida cotidiana y su espacio-temporalidad (187-210). Barcelona: Anthropos.

Rodríguez, E. (2000) Geografia conceptual. Bogotá: Tercer Mundo Editores.

Tuan, Y. (2007). Topofilia. España: Melusina Editores.

Artículo recibido 21 - 09 - 10. Aprobado $26-11-10$. 